Documentation et bibliothèques

DOCUMENTATION BIBLIOTHËQUES

\title{
Leadership et bibliothéconomie : description d'un programme conçu pour les futurs leaders des bibliothèques du Canada
}

\section{Martin Poirier}

Volume 47, numéro 1, janvier-mars 2001

URI : https://id.erudit.org/iderudit/1032649ar

DOI : https://doi.org/10.7202/1032649ar

Aller au sommaire du numéro

Éditeur(s)

Association pour l'avancement des sciences et des techniques de la documentation (ASTED)

\section{ISSN}

0315-2340 (imprimé)

2291-8949 (numérique)

Découvrir la revue

Citer cet article

Poirier, M. (2001). Leadership et bibliothéconomie : description d'un programme conçu pour les futurs leaders des bibliothèques du Canada.

Documentation et bibliothèques, 47(1), 33-38. https://doi.org/10.7202/1032649ar

Tous droits réservés (C) Association pour l'avancement des sciences et des techniques de la documentation (ASTED), 2001
Ce document est protégé par la loi sur le droit d'auteur. L'utilisation des services d'Érudit (y compris la reproduction) est assujettie à sa politique d'utilisation que vous pouvez consulter en ligne.

https://apropos.erudit.org/fr/usagers/politique-dutilisation/ 


\title{
Leadership et bibliothéconomie : description d'un programme conçu pour les futurs leaders des bibliothèques du Canada
}

\author{
Martin Poirier \\ Responsable du développement des systèmes \\ Université de Sherbrooke
}

Acquérir des habiletés de leadership demeure un défi pour les bibliothécaires qui ont, en général, rarement l'occasion de les développer dans le cadre de leurs études ou de leurs emplois. Or, plus d'un employeur valorise ces habiletés lorsque vient le moment de recruter un candidat ou d'accorder une promotion à un employé. La présente chronique vise à faire découvrir un programme de formation axé sur le leadership et offert chaque année dans l'Ouest canadien.

La cinquième édition du programme donné par le Northern Exposure to Leadership Institute (NELI) ${ }^{1}$ s'est déroulée en octobre 2000 à Emerald Lake en Colombie-Britannique. Le but de ce programme est d'aider les bibliothécaires canadiens à développer, améliorer et mettre en pratique leurs habiletés de leadership afin de les rendre aptes à formuler, articuler et réaliser les changements actuels et futurs nécessités par l'évolution constante du domaine bibliothéconomique du XXIe siècle. Pour cette édition 2000, vingtsix bibliothécaires ${ }^{2}$ provenant de diverses régions du Canada ont été sélectionnés à partir d'un dossier décrivant leurs motivations et leurs points de vue sur le leadership. Cinq animateurs ${ }^{3}$ et huit leaders de la profession, ces derniers participant au programme à titre de mentors et de coanimateurs dynamiques, assuraient le bon déroulement des six jours intensifs de formation.

Cette chronique vise non seulement à présenter le contenu de ce programme, mais elle a surtout pour but de le faire connaître aux bibliothécaires de langue française. Peu connu au Québec du fait, sans doute, qu'il se déroule en anglais dans l'Ouest canadien, le programme NELI mérite qu'on s'y intéresse, car il donne la possibilité à ses participants de rencontrer et de partager intensivement avec des collègues de partout à travers le Canada. II permet d'élargir un réseau de contacts professionnels, de partager une même passion et de nourrir un sentiment de fierté face à la profession de bibliothécaire.

Le programme s'étend sur six jours. Les vingt-six participants sont divisés en quatre équipes et deux mentors sont jumelés à chacune de ces équipes. Les journées débutent tôt et les activités se poursuivent jusqu'en soirée. On y retrouve des exercices de réflexion, des remue-méninges en petit groupe et en réunion plénière, des mises en situation, des activités intérieures et certaines en plein air, des témoignages ainsi que des rencontres individuelles entre les participants et les mentors.

La structure pédagogique du programme couvre trois aspects: l'individu, la profession et l'environnement (considéré dans un sens large, c'est-à-dire social et professionnel). Plusieurs principes théoriques sont présentés, mais le programme se veut dynamique et de nombreuses activités permettent de créer des liens entre la théorie et la pratique ou de susciter une réflexion personnelle chez le participant. Le fait de. partager une vie de groupe intensive génère une énergie particulière entre les participants (sans toutefois tomber dans la thérapie de groupe): c'est l'occasion d'établir des liens et d'élargir un réseau de contacts professionnels. Un autre aspect très stimulant du programme est la communication entre des bibliothécaires de tous les domaines: des bibliothèques publiques, académiques ou spécialisées. ${ }^{4}$

\section{Jour 1 : Introduction}

\section{Histoire, technologies, changements sociaux, évolution de la bibliothéconomie}

\author{
Ernie Ingles \\ L'atelier du premier jour a permis de \\ dresser un rapide tableau de l'historique
}

de la profession de bibliothécaire, tout en s'attardant à mettre en contexte la notion de changement dans le domaine de la bibliothéconomie.

Afin de mieux faire ressortir les impacts de la technologie, il importe de situer la profession dans un contexte évolutif. Des éléments perturbateurs tels qu'une nouvelle technologie ou une nouvelle technique finissent par générer un équilibre au sein du travail et amènent la profession à un réajustement.

Dans le processus de propagation des technologies, si certaines sont intégrées et d'autres rejetées, très souvent leur implantation a des impacts sociaux; l'imprimerie, par exemple, a amené les concepts de censure et de propriété intellectuelle.

Avec le développement accéléré des nouvelles technologies, on peut dire que la bibliothéconomie se trouve à la croisée des chemins. Aussi est-il important de se rappeler d'où nous venons et, pour cela,

1. Le NELI a été créé en 1995 par Ernie Ingles, Associate Vice-President (Learning Systems), University of Alberta, et par Donna Brockmeyer-Klebaum, Library Director, St. Thomas More College, University of Saskatchewan. Ingles et Brockmeyer-Klebaum font toujours partie de l'équipe d'animateurs. À l'origine, ce programme s'est inspiré du Snowbird Leadership Institute, un programme américain, mais l'édition canadienne forte de cinq ans d'existence a maintenant ses propres caractéristiques. Voir $<$ http : //www.ualberta.ca/LSS/NELI/>

2. Les participants doivent répondre aux critères de base suivants : avoir terminé leur diplôme de bibliothéconomie depuis moins de 7 ans et avoir au moins 3 ans de pratique professionnelle. Un seul candidat à la fois par année par institution peut être recommandé au programme par la direction de l'institution.

3. Liste des animateurs qui seront cités dans ce compte rendu: Ernie Ingles, Donna Brockmeyer-Klebaum, Karen Adams (Director, Library Services and Information Resources, University of Alberta), Patricia M. Cavill (Library Consultant, Alberta), et Trevor Hamans (Senior Manager, à la retraite).

4. Les sections qui suivent sont en fait des comptes rendus des ateliers dont j'indique pour chacun le nom de l'animateur. Partant du matériel de cours distribué, j'ai d'abord traduit en français les idées principales de chaque atelier pour ensuite réécrire le tout sous forme de compte rendu. 
Tableau 1. Style de leadership du gestionnaire et du leader

\section{GESTIONNAIRE}

administre

est une copie

maintient

se concentre sur les systèmes et les structures

s'appuie sur le contrôle

À une vue étroite

demande comment et quand

À l'œil sur la ligne de fin

imite

accepte le statu quo

est le bon soldat

fait ce qu'il faut faire

Warren, Bennis. Managing people is like herding cats. Provo (US) : Executive Excellence Publishing. 1997. p. 63.

remonter le temps jusqu'au copiste, à l'érudit et à l'ecclésiastique. Dans cette traversée du temps, on remarque que l'on passe du bibliothécaire en tant qu'occupation au bibliothécaire en tant que profession. Tout au long de cette évolution on a vu s'accomplir maintes réalisations. Au $\mathrm{XIXe}$ siècle, on conçoit le rôle du bibliothécaire de différentes façons: d'abord considéré comme un érudit ou comme un administrateur, on lui attribue également un rôle de missionnaire. Au XXe siècle, principalement suite aux changements apportés par l'évolution des technologies, mais aussi à cause des changements sociaux et économiques, on retrouve trois attitudes face à la bibliothéconomie: conservatrice, réformiste et radicale. La venue des nouvelles technologies a apporté des changements sociaux profonds qui nous obligent à repenser ce que signifie être bibliothécaire. Notre façon de réagir à ces changements aura un impact sur la façon dont nous assumerons notre rôle en tant que leader.

\section{Leadership et âge de l'information}

À l'ère de l'information, les leaders en bibliothéconomie se doivent de comprendre la nature d'un environnement fluide, de considérer la profession dans son contexte (social, technologique et historique), de prendre part aux débats et de réagir intelligemment.

\section{LEADER}

\section{innove}

est un original

développe

se concentre sur les gens

inspire confiance

À une perspective étendue

demande quoi et pourquoi

regarde l'horizon

invente

défit le statut quo

est lui-même

fait la bonne chose aiment résoudre les problèmes. Ils font d'autre part un meilleur usage de l'information, ils ont de meilleures habiletés analytiques et ils sont plus tolérants face à l'incertitude.

c) Une autre qualité du leader consiste à passer d'une approche de planification stratégique à une approche de changement stratégique. Avec cette approche, les leaders ont une vision. Ils redéfinissent les modèles traditionnels, ils considèrent le développement stratégique de façon continue et ils travaillent en fonction de buts à long terme tout en explorant différentes manières de les réaliser.

d) Finalement, toujours afin de mieux réagir dans un environnement technologique complexe, les leaders doivent mettre de l'avant une gestion qui s'appuie sur des solutions multiples. Ils expérimentent différentes approches et, pour eux, une erreur n'est pas un échec.

\section{Jour 2 : Leadership: se connaître}

Cette deuxième journée avait pour objectif de présenter aux participants un survol théorique de la notion de leadership et de les amener, par une réflexion sur euxmêmes, à une prise de conscience de leur potentiel de leader et du style de leadership qui les caractérise.

\section{Leadership: comme vous l'aimez}

\section{- Donna Brockmeyer-Klebaum}

Le modèle du leader traditionnel est de rendre la situation des bibliothèques prévisibles, il vaut mieux développer un leadership efficace dans un environnement peu prévisible.

Dans un environnement technologique complexe, les leaders doivent posséder des qualités spécifiques:

a) Ils doivent passer d'une attitude de gestionnaire à celle de leader. Les leaders perçoivent les changements, ils y répondent et savent s'adapter.

b) Les leaders doivent de plus posséder la capacité d'une pensée complexe, c'est-à-dire qu'ils doivent voir les problèmes avec de multiples perspectives et évaluer une panoplie d'options. Les leaders capables d'une pensée complexe une personne forte, active, visionnaire, charismatique: un superleader! Mais il existe d'autres modèles, dont celui du «leader de situation", c'est-à-dire la personne qui, dans certaines situations particulières oriente, supporte, délègue ou guide. On parle aussi du «leader de rotation » qui vient du féminisme, notion reliée non pas au concept de hiérarchie, mais à celui de changement de rôle. Avec les nouvelles approches du leadership, on confère au leader un rôle d'inventeur, de délégué et d'enseignant. Grint ( 1997) présente en détail les courants de pensée sur le leadership. 
Sur ce point, il faut distinguer le gestionnaire et le leader. Voir au Tableau 1 la comparaison du style de leadership entre un gestionnaire et un leader.

Les leaders des bibliothèques doivent avoir un bon jugement politique, une éducation étendue, une grande curiosité, de l'enthousiasme, de la passion, de l'intégrité, une vision, une perception globale de la personne (spiritualité), une tolérance à l'ambiguïté, une capacité de développer des partenariats, une préférence pour l'excellence, pour le raisonnement éthique. Ils doivent également pouvoir définir leurs propres valeurs, apprendre de leurs erreurs, trouver des avantages dans l'adversité, créer le futur, prendre des risques, s'adapter aux changements, prendre des décisions, et, pourquoi pas, savoir célébrer le succès.

II est donc essentiel d'avoir une bonne connaissance de soi pour arriver à un leadership efficace. On y parviendra par différents moyens : écouter sa voix intérieure, évaluer son pouvoir personnel, être en contact et nourrir son âme et son esprit, accepter les responsabilités que l'on est capable d'assumer, apprendre intensivement, faire un retour sur ses expériences, et connaître ses préférences. Pour ce dernier point, un outil tel que le test de personnalité Myers Brigg Type Indicator (MBTI) peut donner de bonnes pistes de connaissance de soi.

\section{Soi en tant que leader et en tant que membre d'une équipe}

\section{Patricia Cavill}

Le Myers-Briggs Type Indicatorest un outil utilisé pendant le programme afin de donner des pistes sur la connaissance de soi-même et sur soi en tant que leader. Le MBTI existe depuis longtemps; basé sur de nombreuses recherches, il se veut constructif et aidant, sans être provocant. Ce genre de test mène à une meilleure connaissance et appréciation des différences individuelles, ce qui est bénéfique sur le plan personnel et professionnel. L'importance de mieux se connaître permet de mieux utiliser ses forces et de diminuer ses faiblesses. Le test permet d'identifier seize catégories de personnes en fonction de quatre facteurs 5 . Connaître ces catégories de personnes peut être utile pour comprendre les autres et mieux communiquer avec eux car cela permet d'apprécier leurs aspects positifs et négatifs.

En parallèle avec le test de $\mathrm{MBTI}$, les participants ont pris part à un jeu de groupe qui demandait une étroite collaboration entre chacun des équipiers afin d'atteindre un but commun. Un crayon feutre au centre d'une armature était soutenu par autant de ficelles qu'il y avait de membres dans l'équipe. Une cible était dessinée sur le plancher et il fallait avoir une coordination de groupe afin que le crayon inscrive une marque dans différentes zones de la cible. Ce jeu utilisait des règles simples pour atteindre des résultats simples, mais comme dans la vie, les changements de règles exigent un ajustement de la part de l'équipe. Le jeu permettait de comprendre les forces et les faiblesses du travail d'équipe.

\section{Leadership: comprendre son environnement}

\section{- Patricia Cavill}

La sensibilisation à la connaissance de son environnement avait pour but d'encourager une attitude pro-active: « $\mathrm{Ce}$ qui importe, ce n'est pas de prédire le futur, mais de cristalliser dans notre esprit ce que nous désirons comme futur 6 ." (Lippitt 1998, 7)

On peut envisager trois manières de réagir face à l'avenir : résister, s'adapter ou inventer. Devenir actif dans la construction de notre avenir ou laisser les autres décider pour nous. Lorsqu'on décide de s'investir, on doit aussi décider comment participer à la création de notre propre futur, que ce soit celui de la société, de son pays ou de sa profession.

Lors d'une séance de remue-méninges, l'ensemble des participants a été amené à identifier les grandes tendances et les facteurs clés. En voici quelques points:

les bibliothécaires doivent être de plus grands défenseurs des bibliothèques et de plus grands communicateurs;

- poser davantage de questions concernant l'argent alloué aux institutions publiques;

s'interroger sur leur pouvoir: patinoire ou bibliothèque publique, qui gagne et pourquoi?;

w problème de droits d'auteur: copyright et technologie; débat sur les données électroniques et la conservation/conversion;

- définition du service personnalisé: quand et où l'usager le veut;

a la bibliothèque pourrait se retrouver comme grande perdante des biens publics;

possibilité de l'augmentation de la présence de l'entreprise privée dans les institutions publiques.

\section{Jour 3 : Leadership: pour un coup de main à la profession}

Cette journée permettait aux participants de comprendre le processus de projection, d'expérimenter un modèle pour définir une vision et d'exprimer une vision pour l'avenir de leur profession.

\section{Qu'est-ce qui fonctionne dans la profession}

\section{Patricia Caville}

L'exercice «fiertés et déceptions" consistait à lister les points de fierté par rapport à la profession et ceux qui nous déçoivent en tant que bibliothécaire. La compilation a permis de faire ressortir six champs de préoccupations. Du côté de la fierté, on retrouvait certains principes professionnels, les innovations et le partage. Du côté des déceptions, les points préoccupants touchaient au profil ou à l'image du bibliothécaire, aux lacunes de la profession ainsi qu'aux priorités.

Cette activité a permis à plusieurs de prendre un certain recul et de constater qu'ils avaient des préoccupations communes. On remarque, entre autres points partagés, le manque de participation des bibliothécaires dans les associations professionnelles, leur engagement à fournir un accès gratuit à l'information à chaque membre de la communauté ou, dans un autre ordre d'idées, la méconnaissance par le public du travail des bibliothécaires et du niveau de leur formation universitaire. «Accepter une situation actuelle telle

\footnotetext{
5. Les quatre facteurs correspondent au type d'énergie (intraverti, extraverti), type de perception, type de jugement, et manière d'aborder le monde. On trouvera dans la littérature beaucoup d'information sur le MBTI.

6. Traduction libre d'une citation donnée en atelier.
} 
qu'elle est ne signifie pas que nous devons abandonner tout espoir d'amélioration et de changement positif dans le futur. Mais cela signifie que l'on doit rester honnête avec soi-même et authentique avec les autres7" (Lippitt 1998, 7). Suite à ce genre d'exercice, il est alors intéressant de réfléchir sur les actions possibles pour améliorer la situation:

\section{Que veut-on pour notre profession?}

«Nous devons décider quel genre de futur nous désirons. Cela nous amène à la difficile question de savoir qu'est-ce que nous valorisons réellement et qu'est-ce que nous voulons vraiment 8 ." (Lippitt $1998,8)$. Un second exercice de remueméninges consistait à réfléchir sur les aspects de la profession à conserver et ceux à éliminer. Les aspects à conserver identifiés par le groupe touchaient à certaines valeurs de base (par exemple, liberté intellectuelle), à l'apprentissage continu, au service (l'exemple de l'accès gratuit) et à l'usager. Des points à éliminer se retrouvaient sous les thèmes de l'estime (mythe par rapport à l'image du bibliothécaire), de la tolérance, des rapports bibliothécaires et usagers, et du contexte technologique (le changement pour le changement).

Les exercices sur nos attentes face à la profession et sur son fonctionnement nous amènent à une prise de conscience actualisée. En fait, ce type d'exercice permet de lier l'apprentissage sur le leadership au quotidien.

Pour agir en leader dans cet environnement, il y a de nouvelles qualités à développer. II faut changer son attitude individuelle: développer une fierté professionnelle, être visionnaire, prendre des risques, accepter le changement, célébrer nos succès et cultiver un esprit d'entrepreneur. II faut également adopter de nouvelles orientations: être plus flexible dans ce que nous faisons et dans la manière de le faire, adopter de nouveaux concepts (ex: une collection de documents numériques n'est plus liée à un lieu physique), repenser le concept de collection et les types de services, augmenter la prise en compte des usagers, être de meilleurs défenseurs de la profession (advocacy). Pour amorcer le changement, on passe inévitablement par la prise de risques. Que le risque génère un aspect positif (un sentiment de pouvoir) ou négatif (peur d'être jugé), on se doit de dépasser ses barrières personnelles.

En conclusion de cette journée de réflexion sur la profession, la notion de changement et de prise de risques, tout le groupe a été amené à réfléchir sur l'avenir de notre profession. Quelle sera la profession en 2010 ? Cette activité a permis la rédaction d'un document qui a été publié dans la revue Feliciter 2001 v.47 no.1. Cette vision propose six rôles pour le bibliothécaire de l'an 2010: enseignant et apprenant, chercheur, activiste pour les droits à l'information, défenseur ou conseiller auprès de la profession, leader dans sa communauté et finalement innovateur.

\section{Jour 4 : Leadership: changement et transition}

Le programme de la quatrième journée aidait les participants à concevoir le changement comme un facteur positif et leur fournissait des outils et des exercices pratiques pour leur permettre de comprendre les notions de transition et de changement.

\section{Éthique : connaître sa profession, se connaître}

\section{Karen Adams}

L'éthique concerne les relations avec les autres et la notion de justice. Sur le plan professionnel, il existe plusieurs codes d'éthique qui spécifient les obligations envers soi-même, les collègues de travail, la communauté des usagers de bibliothèque, les supérieurs et la profession. On peut, par exemple, se référer au code d'éthique de la Canadian Library Association (CLA) ainsi qu'à la Déclaration de la position de la CLA concernant la liberté intellectuelle. Si on s'attarde aux rôles et aux fonctions du bibliothécaire, on pourrait avancer qu'«être bibliothécaire, c'est un acte politique». "L'éthique nous demande d'être conscientisés: faire attention à la manière dont nous agissons et même à la manière dont nous ressentons les cho ses $^{9}$. " (Weston 1999, 30)

\section{Le jeu du chaos: com- prendre les comportements organisationnels et notre rôle par rapport à ceux-ci}

\section{- Trevor Hamans}

Les vingt-six participants ont pris part à un jeu dont le but était de simuler le travail d'équipes de production en usine. II y avait des hauts dirigeants, des cadres intermédiaires et des exécutants. Différentes consignes ont été données, mais avec peu de détails; il fallait chronométrer le jeu. II y a eu beaucoup d'indiscipline, mais l'important était évidemment la réflexion de groupe sur le déroulement du jeu. Le but était de faire comprendre les forces et les faiblesses du travail d'équipe.

\section{Jour 5 : Leadership: vivre en tant que leader}

Cette cinquième journée avait pour objectif de permettre aux participants d'intégrer et de transposer dans la vie quotidienne ce qui avait été vu et de les aider à développer un plan d'action.

\section{Trouver la passion et le courage: défendre une cause}

\section{Patricia Cavill}

Pourquoi la défense ${ }^{10}$ des bibliothèques est-elle nécessaire? Plusieurs réponses sont possibles; principalement, on peut dire à cause des compressions budgétaires et de la diminution des ressources humaines, à cause du grand battage publicitaire autour d'Internet, mais aussi parce que les usagers des bibliothèques font partie d'une majorité silencieuse. D'autre part, il importe de repositionner les bibliothèques dans l'ère de l'information.

Mais qu'est-ce que prendre la défense de la cause des bibliothèques? C'est, par exemple, de développer des activités de relations publiques afin de pro-

\footnotetext{
7. Traduction libre d'une citation donnée en atelier.

8. Traduction libre.

9. Traduction libre d'une citation donnée en atelier

10. Le terme anglais utilisé ici devrait être advocacy (qui ne se traduit pas littéralement en français). J'utilise l'expression «prendre la défense de» faute de mieux.
} 
Tableau 2. Grille «Vous et votre supérieur»

\begin{tabular}{|c|c|c|c|c|}
\hline PROFIL & VOUS & VOTRE SUPÉRIEUR & POINTS COMMUNS & AJUSTEMENTS \\
\hline \multicolumn{5}{|l|}{ Âge } \\
\hline \multicolumn{5}{|l|}{ Sexe } \\
\hline \multicolumn{5}{|l|}{ Style de gestion } \\
\hline \multicolumn{5}{|c|}{ Style de communication } \\
\hline \multicolumn{5}{|l|}{ Niveau de risque } \\
\hline \multicolumn{5}{|c|}{ Attitude face au changement } \\
\hline \multicolumn{5}{|l|}{ Style de présentation } \\
\hline \multicolumn{5}{|l|}{ Style de rapport } \\
\hline \multicolumn{5}{|c|}{ Meilleur moment du jour } \\
\hline \multicolumn{5}{|l|}{ Préjugés } \\
\hline Style d'apprentissage & & & & \\
\hline
\end{tabular}

mouvoir les messages des bibliothèques: dire qui nous sommes, en quoi consiste notre travail, quand et où on le fait, et pour qui. C'est également faire du marketing, c'est-à-dire bien analyser et comprendre les besoins des usagers et adapter nos services en conséquence.

Parmi les besoins signalés dans les recherches, les usagers demandent aux bibliothécaires d'être des accompagnateurs dans ce monde d'information et de savoir en explosion; ils veulent des bibliothèques pour bouquiner et se rencontrer; ils veulent également avoir accès à des documents électroniques plein texte avec graphiques directement lisibles à leur poste de travail.

La défense d'une cause commence souvent par une prise de conscience individuelle pour, par la suite, en convaincre d'autres: il faut donc enrôler! Cette prise de conscience arrive généralement par une sorte de révélation. Par exemple, la re- cherche sur le cancer nous intéresse davantage si un proche en est atteint.

Cependant, défendre une cause est également une question de respect. II faut avant tout comprendre l'environnement dans lequel travaillent les décideurs. Ces derniers doivent composer avec l'humeur changeant du public et la motivation fluctuante des employés ainsi qu'avec l'insatisfaction générale et les préjugés contre les processus administratifs et gouvernementaux.

Notre plus grand défi consiste à trouver les arguments pour convaincre les décideurs. II faut donc mettre en place une stratégie et un plan d'action. Harmoniser notre vision à celle des décideurs tout en poursuivant notre mission d'éducation sont les principaux éléments à considérer. La création d'un programme commun de priorités basé sur les intérêts du public peut s'avérer une tactique efficace.
Voici quelques questions qui pourront nourrir la motivation des défenseurs des bibliothèques: À quel point avez-vous à cœur l'avenir des bibliothèques dans la société de l'information? À quel point prenez-vous à cœur les usagers que vous servez et leur droit d'avoir accès au savoir? À quel point avez-vous à cœur votre profession?

\section{Soyez un leader pour vous- même}

- Donna Brockmeyer-Klebaum

Une introspection est nécessaire pour bien se connaître et pour mieux se percevoir dans sa profession. Il est ainsi intéressant de réfléchir sur les valeurs que nous rattachons à notre travail. La liste peut être très variée: aventure, affiliation, autonomie, défi, créativité, expertise, plaisir, intégrité, leadership, apprentissage, 
argent, passion, reconnaissance, sécurité, estime de soi, service. Quelle est l'essence de la personne que vous êtes en tant que professionnel? Quelles sont vos aspirations professionnelles?

D'un point de vue plus global, nous pouvons nous demander ce que nous attendons de notre organisation ou de notre supérieur. On peut être un leader pour soimême tout en respectant et appuyant son supérieur. Mais pour cela, il faut bien identifier certains aspects de notre milieu. Qu'est-ce que notre supérieur attend de nous en ce qui a trait à la performance, au support, à la loyauté? Quel est son environnement (politique/financier)? Quel est son programme ou son plan d'action? Quelle partie de son plan d'action pouvons-nous faire nôtre? Quelle partie de notre plan d'action peut s'intégrer au sien?

Une simple grille proposée durant l'atelier identifie des points de comparaison à faire entre soi et son supérieur ; elle peut s'avérer un outil efficace pour bien percevoir son rapport avec son supérieur et faire des ajustements judicieux (Voir le Tableau 2. Vous et votre supérieur).

Toujours dans l'optique d'être un meilleur leader pour soi-même, il importe de se positionner dans son environnement immédiat de travail. Quelles responsabilités avez-vous? Êtes-vous un nouvel employé dans ce poste ou avez-vous une longue expérience du travail? Comment êtes-vous appuyé par le personnel? Quelle est votre capacité mais aussi votre désir d'effectuer des changements?

Les rapports de pouvoir occupent une place importante au travail. Du côté du pouvoir personnel, il est sage d'utiliser le pouvoir que l'on a tout en gardant à l'esprit que cela est une question d'expérience, de connaissance, d'expertise, de position, de motivation, de contacts, de politique, et même d'humour... Du côté du pouvoir hiérarchique, il faut obtenir la confiance et l'attention du directeur, car c'est un facteur important pour atteindre des positions de leader.

Être un leader pour soi-même demande une évolution et un dynamisme constants. II faut aller de l'avant pour développer de nouvelles habiletés, participer à des projets spéciaux ou prendre part à des comités, rechercher de nouvelles responsabilités, partager des projets avec quelqu'un dans un nouveau domaine, participer à des programmes continus de dé- veloppement professionnel, obtenir de nouveaux diplômes, assister à des conférences, être actif dans les associations professionnelles, faire du bénévolat. Dans cet élan pour ouvrir les horizons, il faut parler aux professionnels des ressources humaines (suivre l'affichage de nouveaux postes), parler à votre supérieur et aux supérieurs des autres départements, échanger avec votre mentor, consulter votre réseau de contacts. Finalement, pour s'aider à évoluer, il faut aussi identifier les barrières potentielles, par exemple la peur de l'échec, le manque de volonté, d'habiletés ou d'énergie. On peut évidemment faire face à une absence de ressources, d'appui ou simplement un manque de temps.

L'atelier s'est terminé avec l'activité «Vision personnelle: la transformation des barrières » dont les objectifs consistaient à développer à partir des apprentissages du programme NELI un plan d'action à poursuivre à la maison. Un modèle était également présenté pour transformer un obstacle en possibilité d'action, et finalement l'atelier incitait les participants à reconnaître l'importance de consigner ses objectifs. Brièvement, il s'agissait pour chaque participant de penser à un projet qu'il aimerait réaliser dans son poste actuel. Par la suite, en se référant à cette réalisation, il fallait répondre aux questions suivantes: (a) Identifiez les facteurs qui vous aideraient à réaliser votre but; (b) Identifiez le facteur interne principal qui pourrait vous empêcher de réaliser votre but; (c) Identifiez le facteur externe principal qui pourrait vous empêcher de réaliser votre but. Cette étape terminée, l'ensemble du groupe était divisé en petites équipes de six membres et chaque participant présentait son cas pour discussion avec le groupe.

\section{Mot de la fin}

Cette chronique m'a permis de présenter le programme Northern Exposure to Leadership Institute. Même si la part d'apprentissage théorique et informationnel du programme occupe une grande place, elle n'en constitue qu'une seule facette: au-delà de l'apprentissage théorique, le programme NELI s'avère une expérience intense qui permet de développer un réseau de contacts professionnels à travers tout le Canada, et surtout de rendre tangible ce sentiment de fierté d'appartenir à l'effervescente profession de bi- bliothécaire. Pour le leader, on retiendra l'importance de bien se connaître (introspection), d'avoir une pensée articulée sur sa profession, tout en ayant une perception évolutive de son environnement. Passion et évolution continues sont ses principales cartes. Militer, prendre la défense des bibliothèques, être actif dans les associations professionnelles font partie de son plan d'action. Le NELI offre ainsi aux bibliothécaires une occasion unique de parfaire leurs habiletés de leadership et de mieux se positionner dans leur environnement de travail et au sein de leur communauté.

\section{Sources consultées}

Brockmeyer-Klebaum, Donna. 2000. Education to nurture the soul: an interpretive study of a Professional Leadership Institute for Librarians [thèse de doctorat]. Colombie-Britannique: University of British Colombia.

Fisher, Roger and Alan Sharp. 1998. Getting It done. How to lead when you're not in charge. New York: HarperBusiness Book.

Grint, Keith (editor). 1997. Leadership. Classical, contemporary, and critical approaches. New York: Oxford University Press.

Krebs Hirsh, Sandra and Jean M. Kummerow. 1998. Introduction to type in organizations. $3^{\text {nd }}$ édition. Palo Alto, Californie: Consulting Psychologists Press. [Traite de Myers Brigg Type Indicator (MBTI)]

Lee, Susan A. 1994. Leadership: Revised and redesigned for the electronic age, Journal of Library Administration. 20 (2): 1728.

Lippitt, Lawrence L. 1998. Preferred futuring, envision the future you want and unleash the energy to get there. San Francisco: Berrett-Koehler Publishers.

McCabe, Gerard B. and Terrence F Mech. (editors).1998. Leadership and academic librarians. Westport, Connecticut: Greenwood Press, Greenwood library management collection.

Warren, Bennis. 1997. Managing people is like herding cats. Provo (US): Executive Excellence Publishing.

Weston, Anthony. 1999. A practical companion to ethics. New York: Oxford University Press. 